\title{
The common aromatic amino acid biosynthesis pathway is essential in Mycobacterium tuberculosis
}

\author{
Tanya Parish ${ }^{1,2}$ and Neil G. Stoker ${ }^{1} \dagger$
}

Author for correspondence: Tanya Parish. Tel: +44207377 0444. Fax: +44 2072473428. e-mail:t.parish@qmul.ac.uk

1 Department of Infectious and Tropical Diseases, London School of Hygiene and Tropical Medicine, Keppel Street, London WC1E 7HT, UK

2 Department of Medical Microbiology, Barts and the London, Queen Mary's School of Medicine and Dentistry, 32 Newark Street, London E1 2AA, UK

\begin{abstract}
Attempts to construct Mycobacterium tuberculosis strains with a defect in the common aromatic amino acid biosynthesis pathway were made. In other bacteria the genes of this pathway (aro) can be disrupted in the presence of suitable media supplements. The genomic organization of the aro genes in $M$. tuberculosis reveals that there is one operon (aroCKBQ) and three isolated aro genes (aroE, aroG and aroA). The aroK gene was chosen as a target for disruption; this encodes shikimate kinase, which catalyses the fifth step in chorismate biosynthesis. Attempts to replace the wild-type aroK gene with a disrupted allele (aroK $\Delta:$ : hyg) by a two-step homologous recombination procedure were unsuccessful in a wild-type strain. When a second functional copy of aroK was integrated into the chromosome, it was possible to isolate a strain carrying the disrupted gene. Excision of the L5-integrated copy of aroK by the $L 5$ excisionase could be not be achieved in the strain carrying the disrupted copy, but was possible in a strain carrying a wild-type copy. These results demonstrate that the chorismate pathway is essential for the viability of M. tuberculosis.
\end{abstract}

Keywords: chorismate biosynthesis, auxotrophs, gene replacement, bacteriophage L5, excisionase

\section{INTRODUCTION}

Tuberculosis (TB) poses a major worldwide public health problem (Dye et al., 1999). The increasing prevalence of TB, the emergence of multi-drug-resistant strains of Mycobacterium tuberculosis and the devastating effect of co-infection with HIV have highlighted the urgent need for new strategies and tools to control the disease. The available TB vaccine, the bacille Calmette-Guérin (BCG), is an attenuated strain of the closely related organism Mycobacterium bovis. Although widely used, its efficacy has been very variable in clinical trials conducted in different parts of the world (Fine, 2001). A new vaccine and new drugs are urgently needed to combat this devastating disease.

The shikimate biosynthetic pathway, in which 3-deoxyD-arabino-heptulosonate-7-phosphate (DAHP) is con-

\footnotetext{
† Present address: Department of Pathology and Infectious Diseases, Royal Veterinary College, Royal College Street, London NW1 OTU, UK.

Abbreviations: BCG, bacille Calmette-Guérin; DAHP, 3-deoxy-D-arabinoheptulosonate-7-phosphate.
}

verted to chorismate, is required for synthesis of all aromatic amino acids, as well as other important metabolites in bacteria (Fig. 1; Pittard, 1987). Disruption of genes in this pathway has been successfully employed in a wide range of bacterial species to generate strains that are attenuated in models of infection (Gunel-Ozcan et al., 1997; Hoiseth \& Stocker, 1981; Ingham et al., 2002; Simmons et al., 1997; Vaughan et al., 1993). Such strains have been used as live attenuated vaccines (Dilts et al., 2000). With this in mind, the aro A, aroB and aroQ genes were among the first $M$. tuberculosis genes to be cloned and sequenced, and proposed as attractive targets for gene disruption in order to generate a rationally attenuated vaccine to replace BCG (Garbe et al., 1990, 1991).

We and others have demonstrated that M. tuberculosis strains with defects in amino acid biosynthesis pathways show a range of attenuation and can protect in challenge experiments (Hondalus et al., 2000; Smith et al., 2001). One of the genes we attempted to inactivate was aroK (Parish et al., 1999). This gene encodes shikimate kinase which catalyses the fifth step in chorismate biosynthesis, in which shikimic acid is phosphorylated to form 


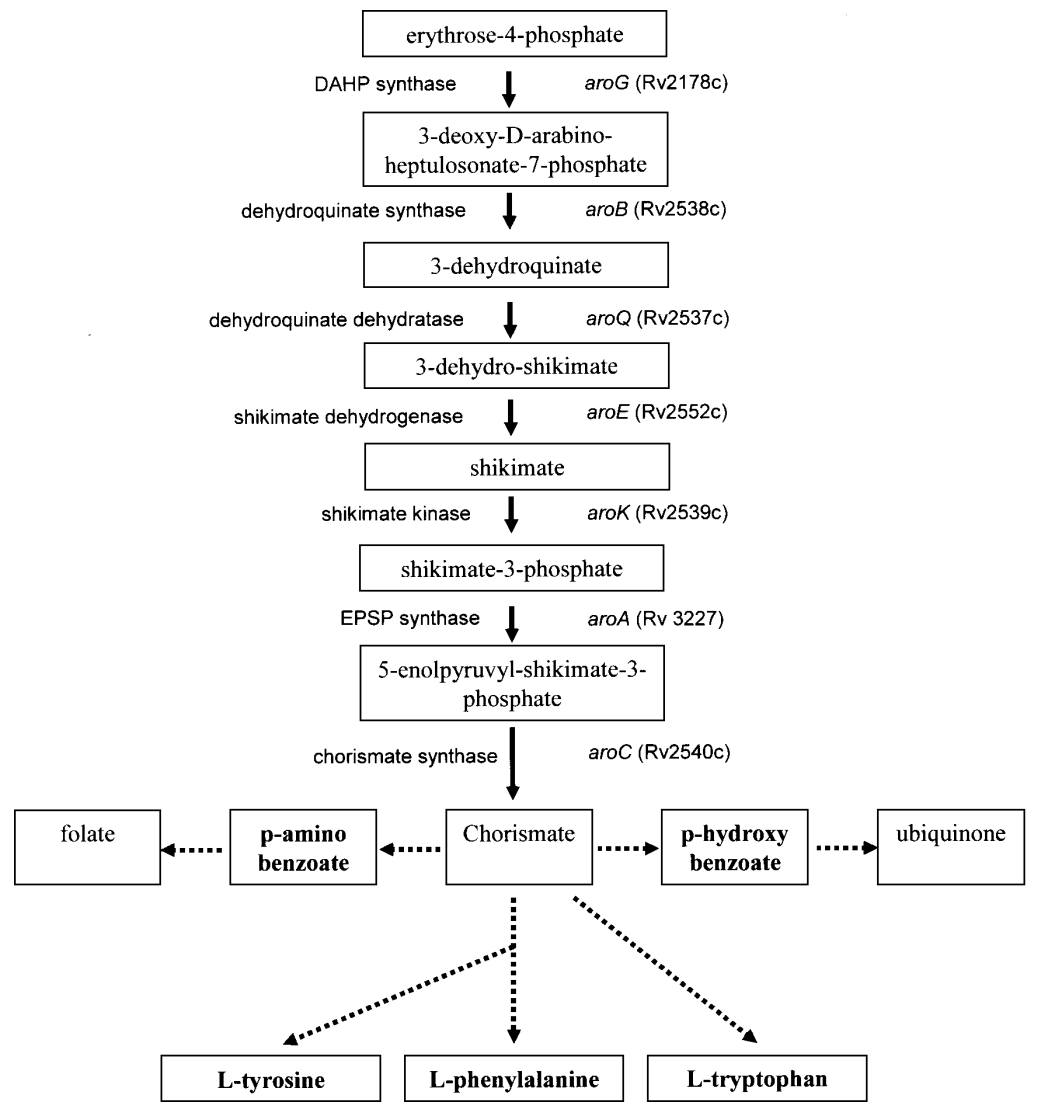

Fig. 1. Schematic outline of chorismate biosynthesis in M. tuberculosis. Enzyme names are given on the left with the corresponding genes on the right. Since there is some confusion over the naming of aro genes, for example aroC (chorismate synthase) has been designated as aroF in the annotated genome, we have also used the Rv numbering system from Cole et al. (1998). Chorismate is used in several biosynthetic pathways, represented by the dotted lines. Components of the aro supplement are shown in bold.

Table 1. Strains and plasmids used

\begin{tabular}{|c|c|c|}
\hline $\begin{array}{l}\text { Strain or } \\
\text { plasmid }\end{array}$ & Description & Genotype/reference \\
\hline \multicolumn{3}{|c|}{$\begin{array}{l}\text { M. tuberculosis } \\
\text { strains }\end{array}$} \\
\hline $\mathrm{H} 37 \mathrm{Rv}$ & Laboratory strain (ATCC 25618) & $\operatorname{aroK}^{+}$ \\
\hline Aroma1 & Single cross-over strain & aroK $K^{+}$kan $P_{\mathrm{hsp} 60^{-}}-s a c B$ aroK $\Delta::$ byg \\
\hline Aroma3 & $\begin{array}{l}\text { Single cross-over strain plus integrating } \\
\text { vector }\end{array}$ & 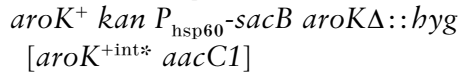 \\
\hline Aroma5 & $\begin{array}{l}\text { Double cross-over strain (mutant) plus } \\
\text { integrating vector }\end{array}$ & $\operatorname{aroK} \Delta:: b y g\left[\operatorname{aroK}^{+\mathrm{int} *}\right.$ aacC1] \\
\hline Aroma6 & Wild-type strain plus integrating vector & $\operatorname{aroK}^{+}\left[\operatorname{aroK}^{+ \text {int* }} \operatorname{aacC} 1\right]$ \\
\hline \multicolumn{3}{|l|}{ Plasmids } \\
\hline pAINT1 & $\begin{array}{l}\operatorname{aro} K^{+} \text {in the integrating vector }-\operatorname{attP} \text { int } \\
\operatorname{aacC1}\end{array}$ & This study \\
\hline pAROB14 & 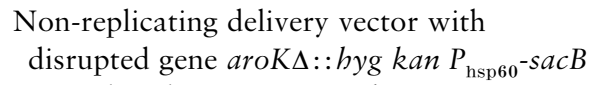 & This study \\
\hline pMV206 & Control replicating vector, $k a n$ & Stover et al. (1991) \\
\hline pJL28 & gp71-xis in pMV206, kan & Parish et al. (2001) \\
\hline
\end{tabular}

*wild-type aroK $\mathrm{K}^{+}$integrated into the chromosome using the L5-based vector. 
shikimate-3-phosphate (Fig. 1). Initial experiments were unsuccessful (Parish et al., 1999) and we demonstrate here that this gene is essential in M. tuberculosis.

\section{METHODS}

Strains and plasmids. M. tuberculosis strains used in this study are shown in Table 1. M. tuberculosis was grown in Middlebrook 7H9 liquid containing 10\% OADC (Becton Dickinson) and $0.05 \% \mathrm{w} / \mathrm{v}$ Tween 80 or on solid Middlebrook 7H10 agar containing $10 \%(\mathrm{v} / \mathrm{v})$ OADC. Kanamycin was used at $20 \mu \mathrm{g} \mathrm{ml} l^{-1}$, hygromycin at $100 \mu \mathrm{g} \mathrm{ml}^{-1}$ and gentamicin at $10 \mu \mathrm{g} \mathrm{ml}^{-1}$ where appropriate. Aro supplement consisted of $40 \mu \mathrm{g} \mathrm{ml}^{-1}$ each of L-tryptophan, L-phenylalanine and $\mathrm{L}$ tyrosine and $250 \mu \mathrm{M}$ each of $p$-hydroxybenzoate, $p$-aminobenzoic acid and 2,3-dihydroxybenzoate.

Bioinformatics. Preliminary sequence data for Mycobacterium smegmatis was obtained from The Institute for Genomic Research website at http://www.tigr.org. Data used were dated 9 Jan 2002. M. bovis and Streptomyces coelicolor sequence data were produced by the M. bovis and S. coelicolor Sequencing Groups at the Sanger Institute and can be obtained from ftp://ftp.sanger.ac.uk/pub/pathogens/mb/ and ftp:// ftp.sanger.ac.uk/pub/S_coelicolor/ respectively.

Construction of vectors. The aroK $\Delta:$ : hyg disrupted allele from plasmid pAROB2 (Parish et al., 1999) was cloned into p2NIL (Parish \& Stoker, 2000a), which does not replicate in mycobacteria, to make pAROB11. This allele has a $324 \mathrm{bp}$ NruI internal fragment of the gene replaced with the byg gene. The vector carries $0.8 \mathrm{~kb}$ of the $3^{\prime}$ end of the $1.2 \mathrm{~kb}$ aroC gene. The marker gene $P_{\text {hsp } 60}-s a c B$ from pGOAL13 (Parish \& Stoker, 2000a) was then cloned into the unique PacI site of pAROB11 to make the final non-replicating delivery vector pAROB14 (Fig. 2b). The wild-type aroK ${ }^{+}$allele (Fig. 2a) was cloned as an EcoRV fragment from cosmid Y159 into pUC-Gm-INT (Mahenthiralingam et al., 1998) which carries the L5 integrase (int) and attachment site $(a t t P)$ and a gentamicin-resistance gene (aacC1), to make the integrating vector pAINT1 (Fig. 2c).

Isolation of single cross-over strain (Aroma1). Approximately $2 \mu \mathrm{g}$ vector DNA (pAROB14) (Fig. 2b) was pretreated with UV light to stimulate homologous recombination and electroporated into M. tuberculosis (Hinds et al., 1999; Parish et al., 1999). Single cross-over strains were selected using hygromycin and kanamycin without aro supplement.

Isolation of double cross-over strains from Aroma1. The single cross-over strain (Aroma1) was streaked out onto media containing aro supplement but without antibiotics and incubated for 2 weeks at $37^{\circ} \mathrm{C}$. A loopful of cells was resuspended in liquid media by vortexing with $1 \mathrm{~mm}$ glass beads. Serial dilutions were plated onto $2 \%(\mathrm{w} / \mathrm{v})$ sucrose plates plus aro supplement (and hygromycin where required). Plates were incubated at $37^{\circ} \mathrm{C}$ for $4-6$ weeks. Sucrose-resistant colonies were then patch-tested for kanamycin and hygromycin resistance on plates containing aro supplement.

Construction of merodiploid strain (Aroma3) and isolation of double cross-over strains. Aroma1 (single cross-over strain) was electroporated with the integrating vector pAINT1. Transformants carrying the vector were selected on gentamicin, kanamycin and hygromycin without aro supplement. An individual colony was picked for further manipulation (Aroma3). Isolation of double cross-over strains was carried out essentially as for the single cross-over strain. Briefly,
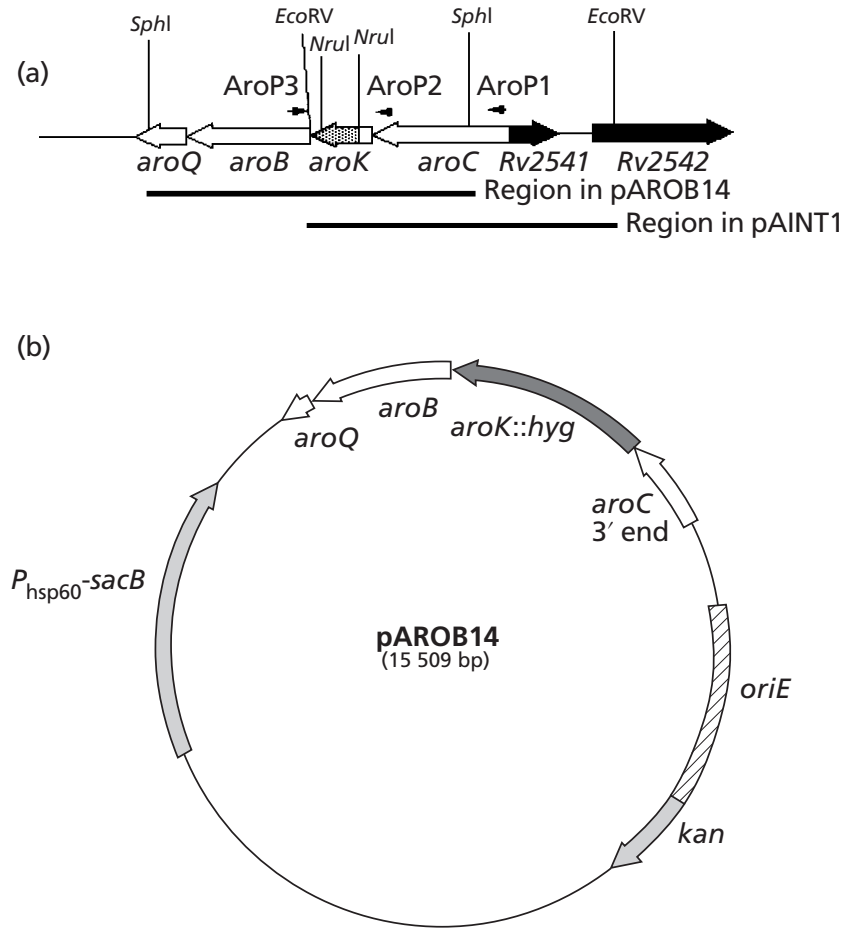

(c)

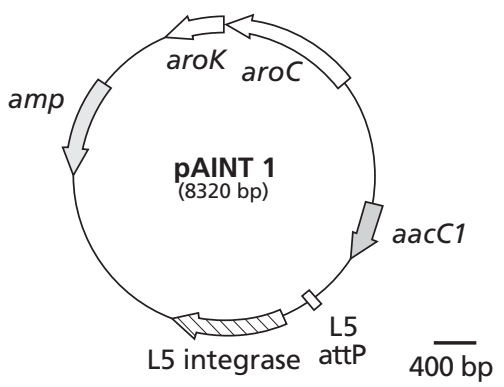

Fig. 2. Plasmid constructs. (a) Arrangement of genes in the $M$. tuberculosis chromosome, showing the regions used in the construction of pAROB14 and pAINT1. Relevant restriction sites are shown. White arrows, aro genes; black arrows, non-aro genes; grey box shows region replaced in aroK. AroP1, AroP2 and AroP3 are PCR primers used for characterizing strains (see Fig. 4). (b) PAROB14 delivery vector used for mutagenesis. The $2.8 \mathrm{~kb}$ Sphl M. tuberculosis fragment indicated in (a) was used to construct the delivery vector. The internal $324 \mathrm{bp} \mathrm{Nrul}$ fragment of aroK was replaced by the hyg gene. (c) pAINT1 vector used to complement mutants. The EcoRV fragment indicated in (a) was cloned into the L5-based integrating vector to make pAINT1.

Aroma3 was streaked out onto media containing aro supplement but without antibiotics and incubated for 2 weeks at $37^{\circ} \mathrm{C}$. A loopful of cells was resuspended in liquid media by vortexing with $1 \mathrm{~mm}$ glass beads. Serial dilutions were plated onto $2 \%(\mathrm{w} / \mathrm{v})$ sucrose plates plus aro supplement (and hygromycin where required). Plates were incubated at $37^{\circ} \mathrm{C}$ for 4-6 weeks. Sucrose-resistant colonies were then patchtested for kanamycin and hygromycin resistance on plates containing aro supplement. 
Excision of L5-integrated plasmids. Aroma5 and Aroma6 strains were electroporated with $0.5 \mu \mathrm{g}$ plasmid pJL28 and plated onto kanamycin plus aro supplement to select for cells carrying the xis plasmid (Parish et al., 2001). Kanamycinresistant colonies were then patch-tested for gentamicin resistance to determine whether the integrated vector was still present.

\section{RESULTS}

\section{Genetic organization of the aro genes}

Analysis of the complete genome sequence of $M$. tuberculosis reveals the presence of seven aro genes predicted to be involved in the chorismate biosynthetic pathway (Cole et al., 1998) (Fig. 1). Four of these (aro CKBQ; Rv2540c-Rv2537c using the $\mathrm{Rv}$ numbering system from Cole et al., 1998) appear to be organized in an operon (Fig. 3) as they are found very close together; aro $C$ and aroK are separated by three bases whereas aroK/aroB and $\operatorname{aroB} / \operatorname{aro} Q$ have overlapping start and stop codons indicating that it is highly likely that they are co-transcribed. aroE $(R v 2552 c)$ is located 10 genes upstream of this operon, possibly in another operon with apparently unrelated genes. The other two aro genes are found elsewhere in the chromosome; aro $A$
( $R v 3227)$ appears to be in an operon with a gene of unknown function $(R v 3228)$ and $\operatorname{aroG}(R v 2178 c)$ is probably monocistronic (Fig. 3a).

A comparison with other mycobacteria shows that the location and organization of aro $A$ and aroG are conserved among the $M$. tuberculosis complex $(M$. tuberculosis and M. bovis), Mycobacterium leprae (Cole et al., 2001) and M. smegmatis, but that the main aro operon is arranged differently (Fig. 3b). In M. leprae, aroE is part of the main operon, with the 10 intervening genes seen in M. tuberculosis missing. In M. smegmatis, aroE is separated from aro $C$ by a single gene, encoding a putative membrane protein. However, unlike $M$. tuberculosis and $M$. leprae, aroQ is not part of this operon and is located elsewhere on the chromosome (Fig. 3c). The related actinomycete S. coelicolor has a similar arrangement to $M$. smegmatis, with aro $Q$ being located separately, although it is flanked by different genes (not shown).

There is some confusion over the nomenclature of the aro genes. We have designated the chorismate synthase $(R v 2540 c)$ gene as aroC to avoid confusion (it is described as aroF in the original Sanger Centre annotation), since it is annotated as aro $C$ in other bacteria

(a)

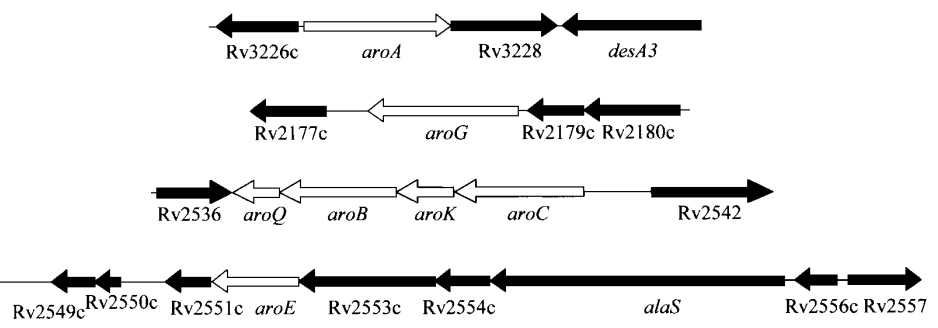

(b)

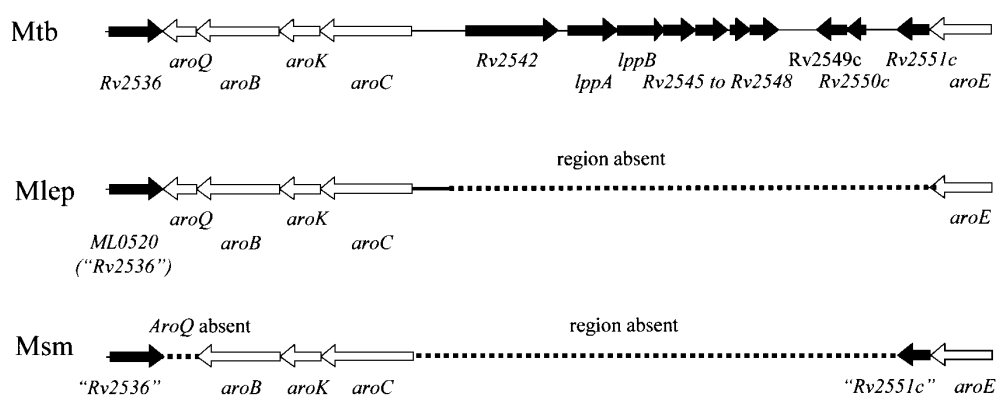

(c)

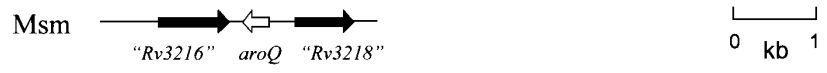

Fig. 3. Genetic organization of the aro genes in mycobacteria. White arrows, aro genes; black arrows, non-aro genes. (a) The location and arrangement of the seven aro genes in $M$. tuberculosis. (b) Organization of the region between aro $E$ and aroC in $M$. tuberculosis (Mtb), $M$. leprae (Mlep) and $M$. smegmatis (Msm). The dotted lines indicate missing regions. (c) The location of the aroQ gene in $M$. smegmatis. Genes representing homologues of the M. tuberculosis genes are indicated in quote marks. 


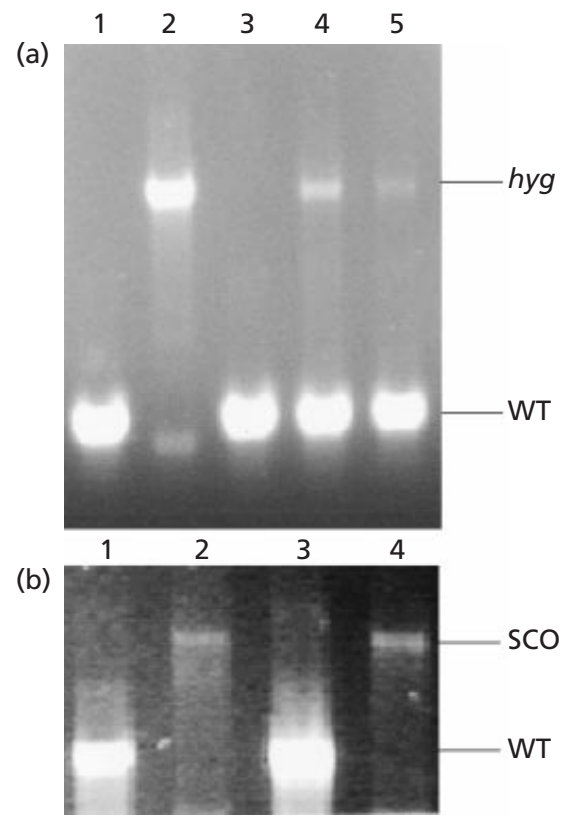

Fig. 4. $P C R$ analysis of strains. (a) Analysis of single and double cross-over strains. PCR was carried out using primers AroP2 and AroP3, which flank the hyg insert in aroK and should amplify both the wild-type locus $(0.5 \mathrm{~kb})$ and the disrupted allele aroK::hyg $(1.7 \mathrm{~kb})$. However, they will not amplify the region in pAINT1 carrying the integrated copy, as AroP3 lies outside the region used for complementation. Lanes: 1, H37Rv; 2, Aroma5; 3, Aroma6; 4, Aroma3; 5, Aroma1. (b) Mapping of single cross overs (SCO). PCR was carried out using primers AroP1 and AroP3. AroP1 lies in aroC outside the insert used in the mutagenesis, while AroP3 lies in $\operatorname{aro} B$, which is within this region, but on the far side of the mutagenized aroK (see Fig. 2). This thus differentiates between SCOs depending on whether the wild-type (WT) or the mutant gene is adjacent to AroP1. Amplification of the WT strain produced a $1.5 \mathrm{~kb}$ fragment, while amplification of the SCO produced a $2.7 \mathrm{~kb}$ band, indicating that the SCO had taken place on the right of aroK as depicted in Fig. 2. Note that the primers will not amplify the region in pAINT1 carrying the integrated copy, as AroP3 lies outside the region used for complementation. Lanes: 1, H37Rv; 2, Aroma5; 3, Aroma6; 4, Aroma3.

and in the well-characterized Escherichia coli pathway, aroF encodes one of the three DAHP synthases. The dehydroquinate synthase gene $(R v 2537 c)$ is designated as aroD in the annotation, but we prefer aro $Q$ as named in the original characterization (Garbe et al., 1991) to reflect the fact that it has homology to fungal catabolic 3 -dehydroquinases $(\operatorname{aro} Q)$ rather than the prokaryotic biosynthetic 3-dehydroquinases (aroD).

\section{Construction of a single cross-over strain (Aroma1)}

We were interested in constructing mutants of $M$. tuberculosis for the aro pathway, since in other bacteria mutants are attenuated (Gunel-Ozcan et al., 1997; Hoiseth \& Stocker, 1981; Ingham et al., 2002; Simmons et al., 1997; Vaughan et al., 1993). We have previously attempted to construct aroK double cross-over strains where the chromosomal copy is replaced by a hygromycin-disrupted allele using a one-step strategy (Parish et al., 1999). This was unsuccessful and demonstrated that there was a low frequency of recombination at this locus (Parish et al., 1999). We therefore changed to a two-step strategy (Parish \& Stoker, 2000a) and tried to isolate double cross-over strains from a single cross-over strain. A two-step strategy is more efficient when there is a low frequency of homologous recombination at a locus, since only one single cross-over is required at each step.

pAROB14 (Fig. 2) was electroporated into M. tuberculosis and single cross-over strains selected using hygromycin and kanamycin. Using $2 \mu \mathrm{g}$ DNA, only one single cross-over colony was obtained. PCR analysis (Fig. 4) and Southern blotting confirmed that it had the single cross-over genotype and demonstrated that recombination had occurred within the shorter $5^{\prime}$ region of homology $(1.0 \mathrm{~kb}$ as compared to $1.5 \mathrm{~kb})$. In our previous work we had obtained a single cross-over strain where recombination had taken place within the longer 3' region (Parish et al., 1999). This showed that recombination could occur on either side to generate viable strains. This strain (Aroma1) is not an aro mutant since it could be grown in the absence of aro supplement.

\section{Second step selection for double cross-over strains from Aroma1}

The single cross-over strain (Aroma1) was then used to try to isolate double cross-over strains. Aroma1 was streaked onto a fresh agar plate (containing aro supplement, but in the absence of antibiotics) to allow the second cross-over to occur. Since the delivery vector contained $P_{\mathrm{hsp} 60}-s a c B$, the strain is sensitive to sucrose. Double cross-over strains can lead to wild-type or mutant cells, depending on the location of the second cross-over. We therefore plated the cells onto plates containing sucrose, hygromycin and aro supplement to select for the double cross-overs leading to the mutant allele. This confirmed that the strain was sucrosesensitive since there was a reduction of approximately $10^{4}$ c.f.u. on plates containing sucrose as compared to those without sucrose. Colonies were then tested for kanamycin resistance to distinguish double cross-overs $\left(k a n^{\mathrm{S}}\right)$ from spontaneous sucrose-resistant $\left(k a n^{\mathrm{R}}\right) \mathrm{mu}-$ tants. Sixty-seven colonies were tested and all were spontaneous sucrose-resistant, single cross-over strains. Thus, no mutant double cross-over strains were isolated. The absence of any mutants using this method suggested that the gene is essential.

We repeated the sucrose selection step in the absence of hygromycin to confirm that homologous recombination was occurring at this locus at a frequency that could be observed. Of 40 sucrose-resistant colonies tested, 32 were spontaneous sucrose-resistant strains $\left(\right.$ kan $^{\mathrm{R}}$ byg $\left.\mathrm{g}^{\mathrm{R}}\right)$ and eight were wild-type double cross-overs $\left(\mathrm{kan}^{\mathrm{s}}\right.$, $\left.h y g^{\mathrm{S}}\right)$. None were mutant double cross-overs $\left(\mathrm{kan}^{\mathrm{s}}\right.$, byg $\left.{ }^{\mathrm{R}}\right)$. This confirmed that we were seeing the second 
(a)

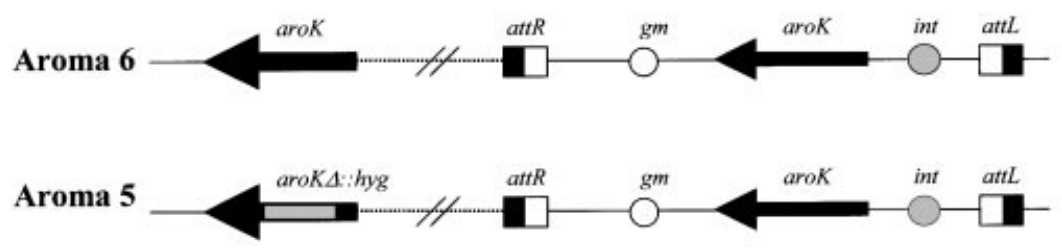

(b)
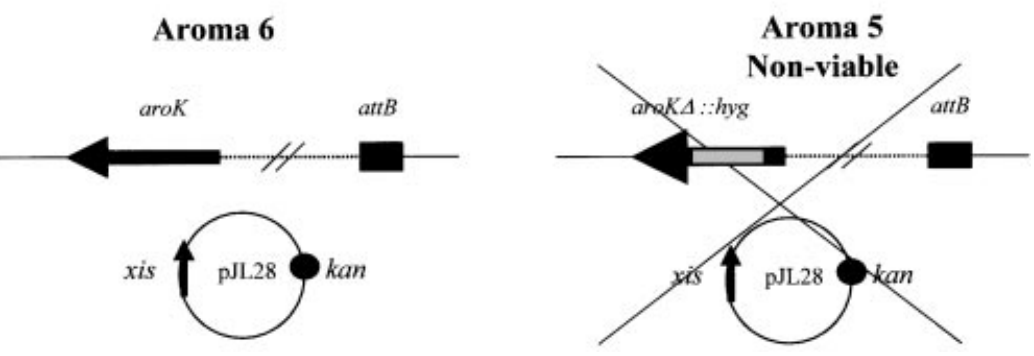

Fig. 5. Excision of L5-integrated plasmids. (a) The genotype of the two strains with respect to aroK is shown. Each strain was transformed with the excisionase plasmid pJL28. (b) The expected transformants for each strain after electroporation with the replicating excisionase plasmid are shown. In each case, the L5-integrated vector should be lost. The attB integration site and the two sites present when a vector is integrated (attL and attR) are shown. kan, kanamycinresistance gene; hyg, hygromycin-resistance gene; gm, gentamicin-resistance gene; xis, L5 excisionase gene; int, L5 integrase gene.

cross-over events, so the absence of mutants was not due to a complete lack of homologous recombination.

\section{Construction of a merodiploid strain (Aroma3) and second step selection for double cross-over strains}

We had already demonstrated the essentiality of another gene, $g \ln E$, by generating a merodiploid strain, which demonstrates that the lack of mutants isolated is not due to the lack of cross-overs leading to mutants, but rather to the viability of the mutants (Parish \& Stoker, 2000b). We used a similar strategy to determine whether aroK is essential. First, we constructed a merodiploid strain (Aroma3) from the single cross-over strain Aroma1, which carried a second wild-type copy of the gene in a different region of the chromosome. An L5 phage-based integrating vector carrying an intact copy of aroK (pAINT1; Fig. 2) was electroporated into Aroma1. The construct was designed to carry the upstream region of aroC as well and therefore contained the natural promoter of the operon. Gentamicin-resistant colonies were isolated and the presence of the integrating plasmid confirmed by Southern analysis. We then plated Aroma3 onto sucrose plates to isolate double cross-over strains as we had done previously with Aroma1. No hygromycin was included, so that both wild-type and mutant double cross-over strains could be isolated. Out of 40 sucrose-resistant colonies tested, nine were wild-type double cross-over strains, one was a mutant double cross-over strain and the remaining 30 were spontaneous sucrose-resistant mutants (single cross-overs). The mu- tant (Aroma5) was analysed by Southern blotting showing that in the mutant double cross-over strain, the wild-type $\operatorname{aroK}$ allele had indeed been replaced, whilst the L5-integrated copy was retained. These results demonstrated that homologous recombination was occurring at this locus to obtain both double cross-over strains although the frequency to give rise to the disrupted allele was low.

\section{Excision of L5-integrated plasmid from double cross- over strain}

Since the number of double cross-over strains obtained was very low in both experiments as compared to the background of spontaneous sucrose resistance, the results were not statistically significant. The bias of the second recombination event towards the wild-type strains means that obtaining enough double cross-over strains to obtain statistical significance would be extremely difficult and laborious. We therefore used a second approach to confirm that the gene was indeed essential (Fig. 5), in which the L5-integrated plasmid is efficiently excised using the L5 excisionase gene (Parish et al., 2001).

We compared two strains; Aroma5 (aroKs::byg aroK $K^{+ \text {int }}$ acc 1 ), described above, and a control strain Aroma6 (aroK ${ }^{+}$aroK ${ }^{+ \text {int }}$ aacC1). We transformed both strains with plasmid pJL28, which replicates in mycobacteria and expresses the L5 exisionase gene xis. These cells were plated onto kanamycin and gentamicin to 
Table 2. Transformation efficiencies

Strains were electroporated with $0 \cdot 5-1 \cdot 0 \mu \mathrm{g}$ plasmid DNA. The number of transformants per microgramme of vector DNA is given.

\begin{tabular}{|lcccc|}
\hline \multirow{2}{*}{ Plasmid } & \multicolumn{3}{c|}{ Strain } \\
\cline { 2 - 5 } & \multicolumn{2}{c|}{ Aroma 6} & \multicolumn{2}{c}{ Aroma 5 } \\
\cline { 2 - 5 } & Kanamycin & $\begin{array}{r}\text { Kanamycin } \\
\text { Gentamicin }\end{array}$ & Kanamycin & $\begin{array}{r}\text { Kanamycin } \\
\text { Gentamicin }\end{array}$ \\
\cline { 2 - 5 } pMV206 & $1 \times 10^{6}$ & $1 \times 10^{6}$ & $1 \times 10^{6}$ & $1 \times 10^{6}$ \\
pJL28 & $1 \times 10^{6}$ & $2 \times 10^{3}$ & $2 \cdot 7 \times 10^{3}$ & $2 \cdot 4 \times 10^{3}$ \\
\hline
\end{tabular}

select for strains carrying both the excisionase and L5integrated plasmids (Table 2 ). We had previously shown that transforming pJL28 into a strain carrying an L5integrated vector results in a $10^{3}$-fold reduction in cells carrying the L5-integrated vector. The small number of survivors are cells in which rearrangements have occurred, for example to inactivate the xis gene (Parish et al., 2001). Thus, when pJL28 is transformed into Aroma6, excision of the integrating plasmid will lead to loss of one of two functional aroK genes and should not affect growth. We therefore expect that the transformation efficiency will be comparable to a control replicating vector, but that only 1 in 1000 transformants will retain the $\mathrm{L} 5$-integrated vector. However, if aroK is essential, in Aroma5 we should see a $10^{3}$-fold reduction in transformation efficiency using pJL28, as all cells in which the L5-integrated plasmid is excised will die and all the transformants should retain the L5-integrated vector.

Table 2 shows the results from the transformations. As expected, the efficiency of transformation of Aroma6 with pJL28 was comparable to the control vector pMV206. When plated onto kanamycin plus gentamicin, the number of colonies was $10^{3}$-fold lower, indicating that virtually all cells containing pJL28 had lost the integrating plasmid. With Aroma5, the transformation efficiency with the control plasmid was normal, but was reduced $10^{2}$ - to $10^{3}$-fold with pJL28 ( $P$ value using Fisher's exact test $\left.<10^{-126}\right)$. To confirm that the small number of surviving colonies with Aroma5 still contained the L5-integrated plasmid, 24 kanamycin-resistant transformants from each pJL28 transformation were patch-tested for gentamicin resistance. All Aroma6 transformants $(24 / 24)$ were sensitive, while all Aroma5 transformants $(24 / 24)$ were resistant, showing efficient excision of the L5-integrated vector from Aroma6 but not Aroma5. This confirms the essentiality of aroK, as $99.9 \%$ of transformants did not survive, and analysis showed the others still contained aroK.

\section{DISCUSSION}

The fact that we were unable to isolate strains containing only a disrupted allele of aroK demonstrate that this gene is essential for viability of M. tuberculosis. The construction of a merodiploid strain containing an L5integrated copy of aroK followed by use of the L5 excisionase allowed us to provide formal proof that the aroK gene is essential under the conditions employed. That is, homologous recombination leading to gene disruption could only occur in the presence of a functional copy of the gene and in such strains the L5integrated copy could not be excised. The failure to obtain any aro mutants of $M$. tuberculosis had previously been attributed to the difficulty of obtaining successful gene replacement at all in slow-growing mycobacteria. We have now shown that in fact it is because the common aromatic amino acid biosynthesis pathway is essential.

We have used both one- (Parish et al., 1999) and twostep recombination strategies to construct a mutant strain carrying a disrupted allele of aroK. In each approach, the disrupted allele was the same, but the delivery vector backbone was different. Using either vector we have seen a very low frequency of recombination as compared to other loci. Previous work has demonstrated that the frequency of recombination does vary greatly between loci (Parish et al., 1999) and these data support the hypothesis that there is an inherently low frequency of recombination at the aroK locus. It is unlikely that the low frequency reflects an inability to get plasmids into the cells as the transformation efficiency using a replicating plasmid is $10^{7}$ per $\mu \mathrm{g}$ DNA.

The possibility that the aroK $\Delta:$ : byg mutation results in a polar effect on downstream genes can be discounted. The aroK gene is found in a cluster of four closely linked shikimate pathway genes with the structure aroCaroK-aroB-aroQ, which probably form a single transcription unit. Although the insertion of the byg gene may interrupt transcription from the natural promoter, the $a r o B$ and aro $Q$ genes should still be expressed from the $h y g$ promoter. This is confirmed by the fact that the region used in the complementing integrated vector contained aro $C$ and aroK, but not the two downstream genes. Thus the inability to obtain gene replacement cannot be attributed to disruption of the function of aroB and/or aroQ.

The end product of the common aromatic amino acid pathway is chorismate, the precursor for biosynthesis of 
the aromatic amino acids tyrosine, tryptophan and phenylalanine, as well as folate and ubiquinone (Fig. 1). We expected that the aromatic supplements used would be sufficient to permit the growth of aroK mutants, as it is in other bacteria that have been studied. Fig. 1 shows the supplements added to the media in relation to the pathway. Several possibilities exist to explain the essentiality of this pathway. The first is that the supplements are not transported into the cell. We have already isolated a tryptophan auxotroph, which can be grown in the presence of L-tryptophan, showing that this amino acid is transported into the cell (Parish et al., 1999). M. tuberculosis has a homologue of AroP2, an aromatic amino acid transporter (Cole et al., 1998), so we would expect that the other aromatic amino acids are transported into the cell as well.

The second possibility is that M. tuberculosis relies on a constant supply of the final product of one or more downstream pathway(s) for its survival. It is feasible that there is another, as yet uncharacterized, branch from the aromatic pathway in mycobacteria. The end product of this pathway would have to be added to the supplements in order for aroK mutants to be viable. We also tried to isolate mutants in the presence of chorismate supplementation, but again were unsuccessful, suggesting that a branch point after chorismate itself is unlikely.

There are several differences between the wellcharacterized E. coli genes and the M. tuberculosis homologues. The first step of the pathway is catalysed by three enzymes in E. coli, each of which is independently regulated, whereas $M$. tuberculosis possesses only one DAHP synthase $(\operatorname{aroG})$. E. coli also has two shikimate kinase genes (aroK and aroL), whilst $M$. tuberculosis only has one. Therefore there appears to be less redundancy in the M. tuberculosis pathway. Vinella et al. (1996) identified a second function for the E. coli aroK gene product that is distinct and unrelated to its shikimate kinase activity. Should the M. tuberculosis enzyme possess a similar activity it is possible that disruption of this activity would explain the inability of the bacteria to grow in the absence of a functional copy of the gene.

Analysis of the genetic organization of the aro genes between slow- and fast-growing mycobacteria reveals differences in the main aro operon. This may reflect differences in the regulation or the function of the pathway. We do not know if the common aromatic amino acid pathway is essential in all mycobacteria or whether this phenomenon is confined to M. tuberculosis alone.

The genes and pathways that are essential for the growth of $M$. tuberculosis make attractive drug targets since inhibiting their function may kill the organism. The chorismate pathway is one of great interest in that it makes a good target for drug development. The pathway is absent from the human host, thus the products cannot be gleaned from the host environment and inhibition of any of the mycobacterial enzymes is unlikely to have a toxic side effect on the host. The evidence that this pathway is essential for M. tuberculosis even in the presence of exogenous supplements reinforces its attractiveness as a drug target. It is interesting to note that the M. tuberculosis shikimate kinase (AroK) has been crystallized (Gu et al., 2001) and the structure of the dehydroquinase (AroQ) has been determined (Gourley et al., 1999), opening the possibility of rational design of inhibitors.

\section{ACKNOWLEDGEMENTS}

Tanya Parish was partly funded by the Glaxo Wellcome Action TB Initiative. We thank Ken Duncan for helpful comments. Sequencing by TIGR of the M. smegmatis genome was accomplished with support from NIAID.

\section{REFERENCES}

Cole, S. T., Brosch, R., Parkhill, J. \& 39 other authors (1998). Deciphering the biology of Mycobacterium tuberculosis from the complete genome sequence. Nature 393, 537-544.

Cole, S. T., Eiglmeier, K., Parkhill, J. \& 41 other authors (2001). Massive gene decay in the leprosy bacillus. Nature 409, 10071011.

Dilts, D. A., Riesenfeld-Orn, I., Fulginiti, J. P. \& 11 other authors (2000). Phase I clinical trials of aro $A$ aroD and aro $A$ aroD btrA attenuated $S$. typhi vaccines; effect of formulation on safety and immunogenicity. Vaccine 18, 1473-1484.

Dye, C., Scheele, S., Dolin, P., Pathania, V. \& Raviglione, M. C. (1999). Global burden of tuberculosis: estimated incidence, prevalence, and mortality by country. J A M A (J Am Med Assoc) 282, 677-686.

Fine, P. E. M. (2001). BCG : the challenge continues. Scand J Infect Dis 33, 243-245.

Garbe, T., Jones, C., Charles, I., Dougan, G. \& Young, D. (1990). Cloning and characterization of the aroA gene from Mycobacterium tuberculosis. J Bacteriol 172, 6774-6782.

Garbe, T., Servos, S., Hawkins, A., Dimitriadis, G., Young, D., Dougan, G. \& Charles, I. (1991). The Mycobacterium tuberculosis shikimate pathway genes - evolutionary relationship between biosynthetic and catabolic 3-dehydroquinases. Mol Gen Genet 228, 385-392.

Gourley, D. G., Shrive, A. K., Polikarpov, I., Krell, T., Coggins, J. R., Hawkins, A. R., Isaacs, N. W. \& Sawyer, L. (1999). The two types of 3-dehydroquinase have distinct structures but catalyze the same overall reaction. Nat Struct Biol 6, 521-525.

Gu, Y., Reshetnikova, L., Li, Y., Yan, H., Singh, S. V. \& Ji, X. (2001). Crystallization and preliminary X-ray diffraction analysis of shikimate kinase from Mycobacterium tuberculosis in complex with MgADP. Acta Crystallogr D Biol Crystallogr 57, 1870-1871.

Gunel-Ozcan, A., Brown, K. A., Allen, A. G. \& Maskell, D. J. (1997). Salmonella typhimurium aroB mutants are attentuated in BALB/c mice. Microb Pathog 23, 311-316.

Hinds, J., Mahenthiralingam, E., Kempsell, K. E., Duncan, K., Stokes, R. W., Parish, T. \& Stoker, N. G. (1999). Enhanced gene replacement in mycobacteria. Microbiology 145, 519-527.

Hoiseth, H. K. \& Stocker, B. A. (1981). Aromatic-dependent Salmonella typhimurium are non-virulent and effective as live vaccines. Nature 291, 238-239.

Hondalus, M. K., Bardarov, S., Russell, R., Chan, J., Jacobs, W. R. \& Bloom, B. R. (2000). Attenuation of and protection induced by 
a leucine auxotroph of Mycobacterium tuberculosis. Infect Immun 68, 2888-2898.

Ingham, A., Zhang, Y. \& Prideaux, C. (2002). Attenuation of Actinobacillus pleuropneumoniae by inactivation of aroQ. Vet Microbiol 84, 263-273.

Mahenthiralingam, E., Marklund, B. I., Brooks, L. A., Smith, D. A., Bancroft, G. J. \& Stokes, R. W. (1998). Site-directed mutagenesis of the 19-kilodalton lipoprotein antigen reveals no essential role for the protein in the growth and virulence of Mycobacterium intracellulare. Infect Immun 66, 3626-3634.

Parish, T. \& Stoker, N. G. (2000a). Use of a flexible cassette method to generate a double unmarked Mycobacterium tuberculosis tly $A$ plcABC mutant by gene replacement. Microbiology 146, 19691975.

Parish, T. \& Stoker, N. G. (2000b). $g \ln E$ is an essential gene in Mycobacterium tuberclosis. J Bacteriol 182, 5715-5720.

Parish, T., Gordhan, B. G., McAdam, R. A., Duncan, K., Mizrahi, V. \& Stoker, N. G. (1999). Production of mutants in amino acid biosynthesis genes of Mycobacterium tuberculosis by homologous recombination. Microbiology 145, 3497-3503.

Parish, T., Lewis, J. \& Stoker, N. G. (2001). Use of the mycobacteriophage L5 excisionase in Mycobacterium tuberculosis to demonstrate gene essentiality. Tuberculosis 81, 359-364.

Pittard, A. J. (1987). Biosynthesis of the aromatic amino acids. In Escherichia coli and Salmonella typhimurium: Cellular and
Molecular Biology, pp. 368-394. Edited by J. L. Ingraham, K. B. Low, B. Magasanik, M. Schaechter \& H. E. Umbarger. Washington, DC: American Society for Microbiology.

Simmons, C. P., Hodgson, A. L. M. \& Strugnell, R. A. (1997). Attenuation and vaccine potential of aro $Q$ mutants of Corynebacterium pseudotuberculosis. Infect Immun 65, 3048-3056.

Smith, D. A., Parish, T., Stoker, N. G. \& Bancroft, G. J. (2001). Characterization of auxotrophic mutants of Mycobacterium tuberculosis and their potential as vaccine candidates. Infect Immun 69, 1142-1150.

Stover, C. K., Delacruz, V. F., Fuerst, T. R. \& 11 other authors (1991). New use of BCG for recombinant vaccines. Nature 351, 456-460.

Vaughan, L. M., Smith, P. R. \& Foster, T. J. (1993). An aromaticdependent mutant of the fish pathogen Aeromonas salmonicida is attenuated in fish and is effective as a live vaccine against the salmonid disease furunculosis. Infect Immun 61, 2172-2181.

Vinella, D., Gagny, B., JoseleauPetit, D., Dari, R. \& Cashel, M. (1996). Mecillinam resistance in Escherichia coli is conferred by loss of a second activity of the AroK protein. J Bacteriol 178, 3818-3828.

Received 27 March 2002; revised 26 June 2002; accepted 27 June 2002. 